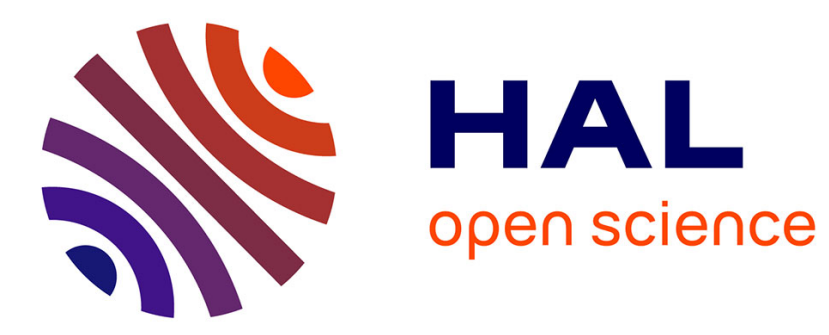

\title{
Technical aspects of the soleil project
}

M.-P. Level

\section{To cite this version:}

M.-P. Level. Technical aspects of the soleil project. Journal de Physique IV Proceedings, 1994, 04

(C9), pp.C9-319-C9-326. 10.1051/jp4:1994955 . jpa-00253517

\section{HAL Id: jpa-00253517 https://hal.science/jpa-00253517}

Submitted on 1 Jan 1994

HAL is a multi-disciplinary open access archive for the deposit and dissemination of scientific research documents, whether they are published or not. The documents may come from teaching and research institutions in France or abroad, or from public or private research centers.
L'archive ouverte pluridisciplinaire HAL, est destinée au dépôt et à la diffusion de documents scientifiques de niveau recherche, publiés ou non, émanant des établissements d'enseignement et de recherche français ou étrangers, des laboratoires publics ou privés. 


\section{Technical aspects of the soleil project}

M.-P. Level

LURE, Bâtiment 209D, Centre Universitaire, 91405 Orsay cedex, France

The SOLEIL project is built around a $2.15 \mathrm{GeV}$ positron storage ring with its own injector, i.e. a linear accelerator and a booster. The scientific community have required a large number of insertion devices optimized in the soft X-ray range and the ultra-violet, several operation modes offering a high brilliance or a usable time structure with a maximum beam lifetime. The choice of the machine energy, of positrons circulating as particles, of the magnetic lattice, and the type of radio-frequency, etc. will be discussed through a more detailed presentation of the machine. Comments will be made about the tolerances required for the beam and the implications for the building stability.

\section{INTRODUCTION}

The main features for new synchrotron radiation light sources based on storage ring are the number of insertion devices, the brilliance, the lifetime, the stability of the photon beams and the flexibility of the optics for the different modes of operation.

It is difficult to define a universal criterion for all kind of sources and all users [1]. In this paper, the different possible goals are discussed and two alternative configurations of the storage ring are presented assuming the same global cost.

\section{STORAGE RING LATTICE}

For a medium energy range machine, the main limitation of the lifetime is the Touschek effect, especially for operation with few intense bunches. The reduction of the energy acceptance $(\Delta \mathrm{P} / \mathrm{P})$ due to the undulator non linear fields, further increases this effect. In order to keep an acceptable lifetime, the particle density should not be too high and consequently the emittance too low.

In other respects, experience acquired on medium energy range machines shows that most of the time, undulators are used with high $\mathrm{K}$ values and constant gap. The effective brilliance is then the average brilliance on and off axis, taking into account the even and odd harmonics. The figure of merit depends then on the beam dimensions which scale as the square root of the emittances. All these considerations and the results of a survey including all the LURE users (150 groups of scientists) led, in a first stage, to retain for the SOLEIL storage ring design the following criteria :

- Polyvalent synchrotron radiation source in the energy range of $20 \mathrm{eV}$ to $20 \mathrm{keV}$ photon beams.

- Excellent lifetime with the two modes of operation, 20 hours for $300 \mathrm{~mA}$ in the multibunch operation, 10 hours for $6 \times 10 \mathrm{~mA}$ bunches in the temporal structure operation.

- Large number of insertion devices (>12). 
- Long straight sections ( $>5 \mathrm{~m}$ ). ACO.

- Large number of beamlines installed $(>30)$ to replace the potentialities of both DCI and Super-

- Optimised cost by minimization of the machine length $(\approx 200 \mathrm{~m})$.

For the required critical wavelength $2.5 \AA\left(\varepsilon_{\mathrm{c}}=5 \mathrm{keV}\right)$ and a $1.6 \mathrm{~T}$ magnetic field, the machine energy is $2.15 \mathrm{GeV}$.

A modified DBA lattice allowing the implementation of two insertion devices per period was chosen. An emittance of $3710^{-9} \mathrm{~m}$.rad meets the lifetime request of the temporal structure operation. This value can be reduced to $1610^{-9} \mathrm{~m}$.rad by ajusting the dispersion fonction in the straight sections. This optics is suitable for the high brilliance multibunch operation mode (see Fig. 1).

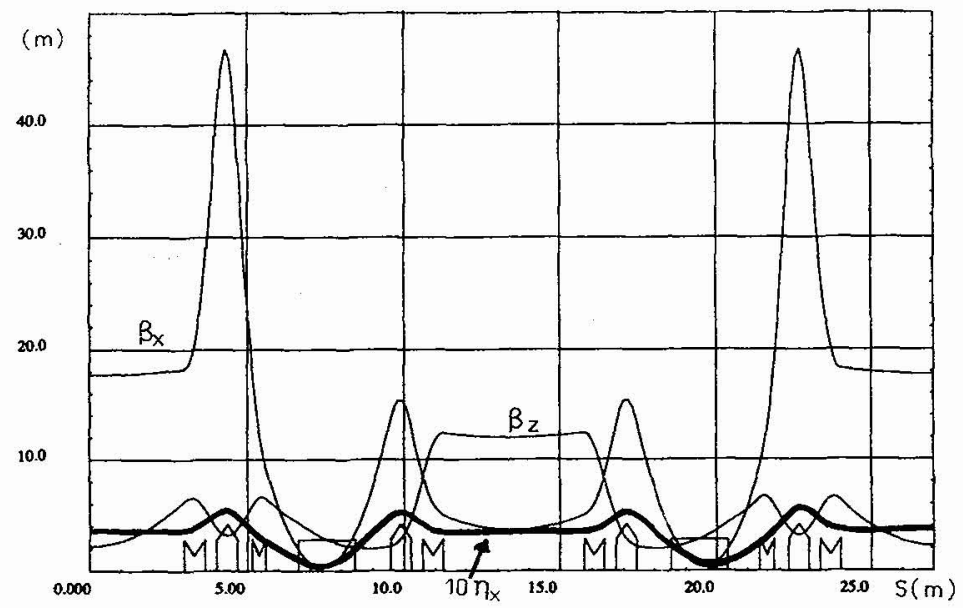

Fig. 1 : Optical functions for modified DBA lattice with $\eta_{\mathrm{X}} \neq 0$ in all straight sections, $\varepsilon_{\mathrm{x}}=1610^{-9} \mathrm{~m} . \mathrm{rad}$.

The resulting storage ring design, $216 \mathrm{~m}$ long, made of 8 periods with 13 insertion devices and 35 experimental beamlines is shown Fig. 2 .

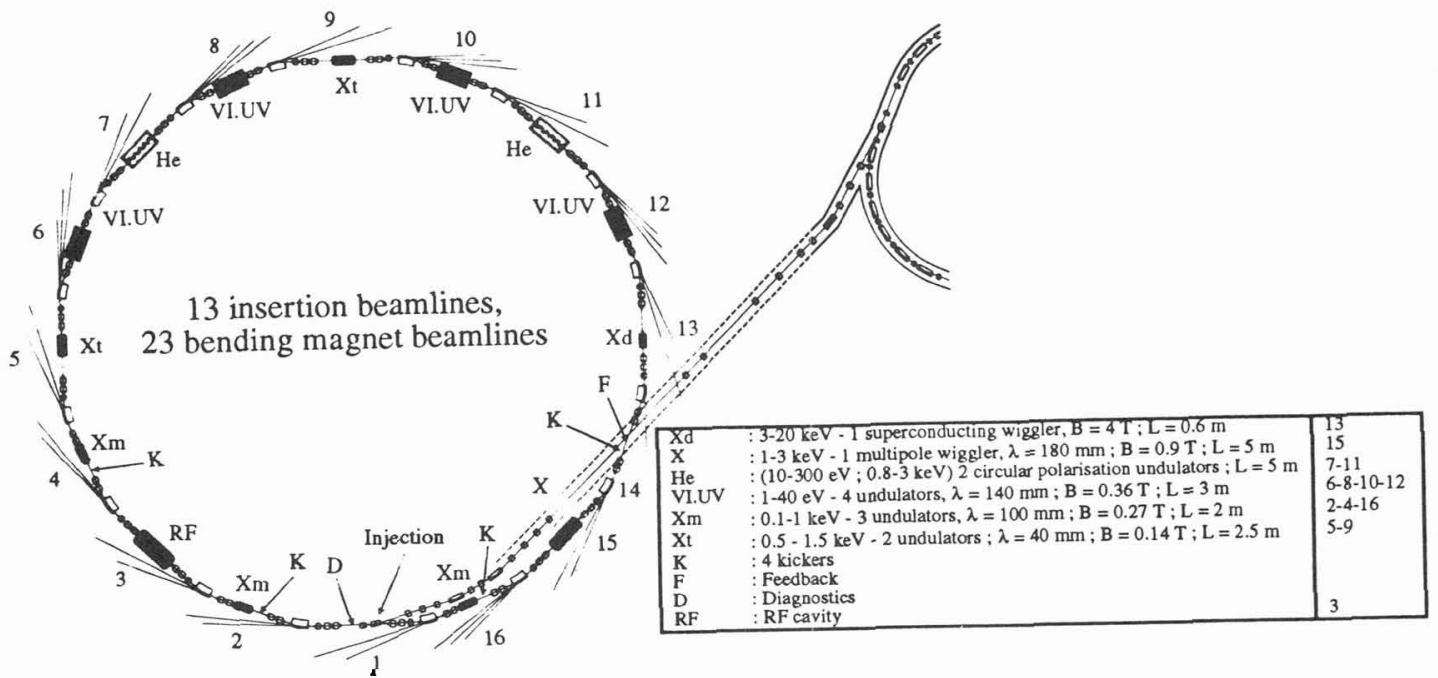

Fig. 2 : Overview of SOLEIL with a typical insertion device distribution. 
The capital cost for the source has been evaluated to $270 \mathrm{MFF}$. It includes : a high current Linac with $200 \mathrm{MeV}$ energy followed by a quarter wavelength converter, a $400 \mathrm{MeV}$ positron Linac and a booster increasing the beam energy to $2.15 \mathrm{GeV}$. The beamlines and experiments budget is $315 \mathrm{MFF}$, components recovered from Super-ACO and DCI reduce this bill by 67 MFF. Taking into account the price of buildings and infrastructures of the new laboratory, the total cost rises to 812 MFF HT (value 1993) [2].

Recently, there was a further request for more flexibility in order to provide two more modes of operation, high brilliance ( $10^{18}$ to $10^{19} \mathrm{phs}^{-1} \mathrm{mrad}^{-2} \mathrm{~mm}^{-2} / 0.1 \%$ from undulator) and free electron laser.

A new storage ring configuration is currently being studied with the same DBA structure but 12 periods providing a minimum emittance of $410^{-9} \mathrm{~m}$.rad tunable to $3010^{-9} \mathrm{~m} . \mathrm{rad}$. The Fig. 3 presents the optical functions of one period for $\varepsilon_{\mathrm{x}}=3.910^{-9} \mathrm{~m} . \mathrm{rad}$.

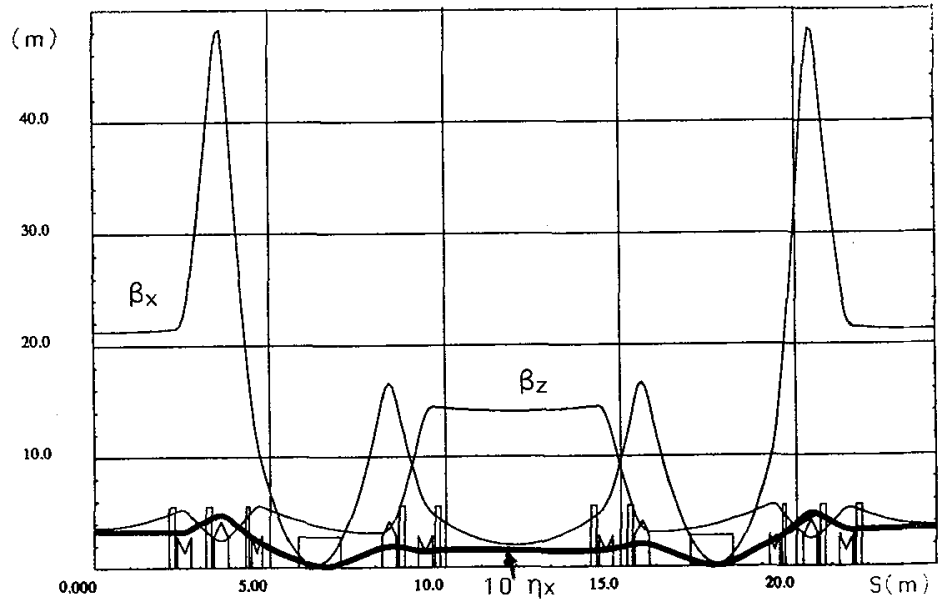

Fig. 3 : Optical functions (1/12 of the machine) $\varepsilon_{\mathrm{x}}=3.910^{-9} \mathrm{~m} \cdot \mathrm{rad}$.

The main parameters are $: v_{x}=15.7 ; v_{z}=9.7 ; \alpha=6.810^{-4} ; \sigma_{E} / E=8.610^{-4}$ and the beam dimensions at the source points are given in the following Table 1.

Table 1

\begin{tabular}{|l|l|c|c|c|c|}
\cline { 3 - 6 } \multicolumn{2}{c|}{} & $\begin{array}{c}\sigma_{\mathrm{x}} \\
(\mu \mathrm{m})\end{array}$ & $\begin{array}{c}\sigma_{\mathrm{x}}^{\prime} \\
(\mu \mathrm{rad})\end{array}$ & $\begin{array}{c}\sigma_{\mathrm{z}} \\
(\mu \mathrm{m})\end{array}$ & $\begin{array}{c}\sigma_{\mathrm{z}}^{\prime} \\
(\mu \mathrm{rad})\end{array}$ \\
\hline $\begin{array}{l}\text { Bending magnet beamlines } \\
\text { (up to 21) }\end{array}$ & Odd & 44 & 124 & 39 & 10 \\
\cline { 2 - 7 } & Even & 63 & 77 & 37 & 10 \\
\hline $\begin{array}{l}\text { Insertion beamlines } \\
\text { (up to 21) }\end{array}$ & Odd & 386 & 13 & 37 & 10 \\
\cline { 2 - 7 } & Even & 142 & 44 & 74 & 5 \\
\hline
\end{tabular}

The whole design (Fig. 4) consists of two superperiods with two $10 \mathrm{~m}$ long straight sections, one of them dedicated to free electron laser [3]. The optimisation to match the optical fonctions between the long straights and the arcs for the same emittance is in progress. 


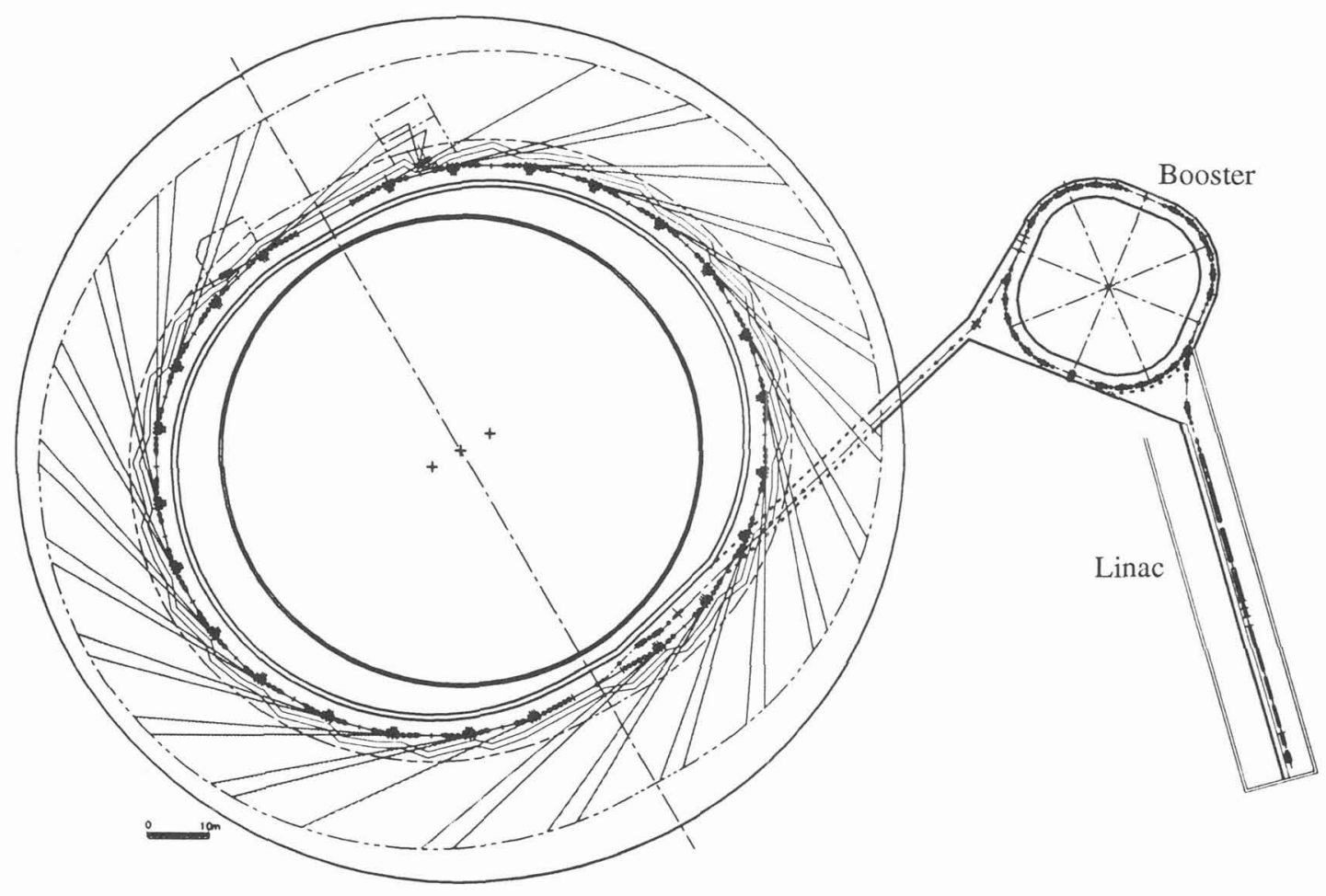

Fig. 4 : Overview of SOLEIL with the new storage ring configuration.

Fig. 5 shows the optical functions of one superperiod for the FEL mode with $\varepsilon_{\mathrm{x}}=1610^{-9} \mathrm{~m} \cdot \mathrm{rad}$ at $1.5 \mathrm{GeV}$. The $\beta_{\mathrm{x}}$ and $\beta_{\mathrm{z}}$ values in the long straight sections have been adjusted to $6 \mathrm{~m}$.

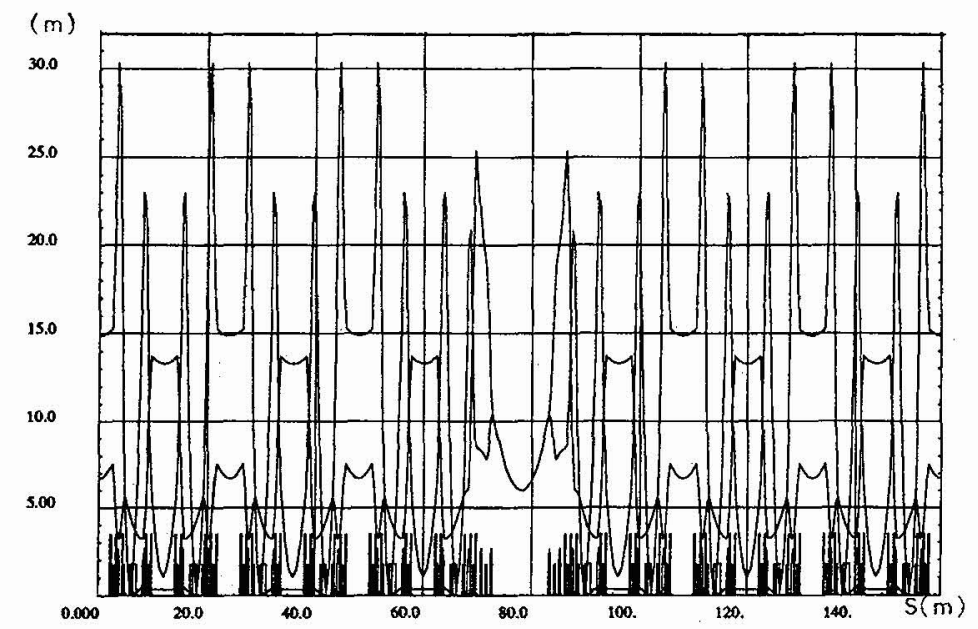

Fig. 5 : Optical functions for one superperiod, $\varepsilon_{\mathrm{x}}=1610^{-9} \mathrm{~m} \cdot \mathrm{rad}$. 
Fig. 6 focuses on FEL straight section and illustrates the possibility of two colour experiments from bending magnet radiation and from an other insertion radiation.

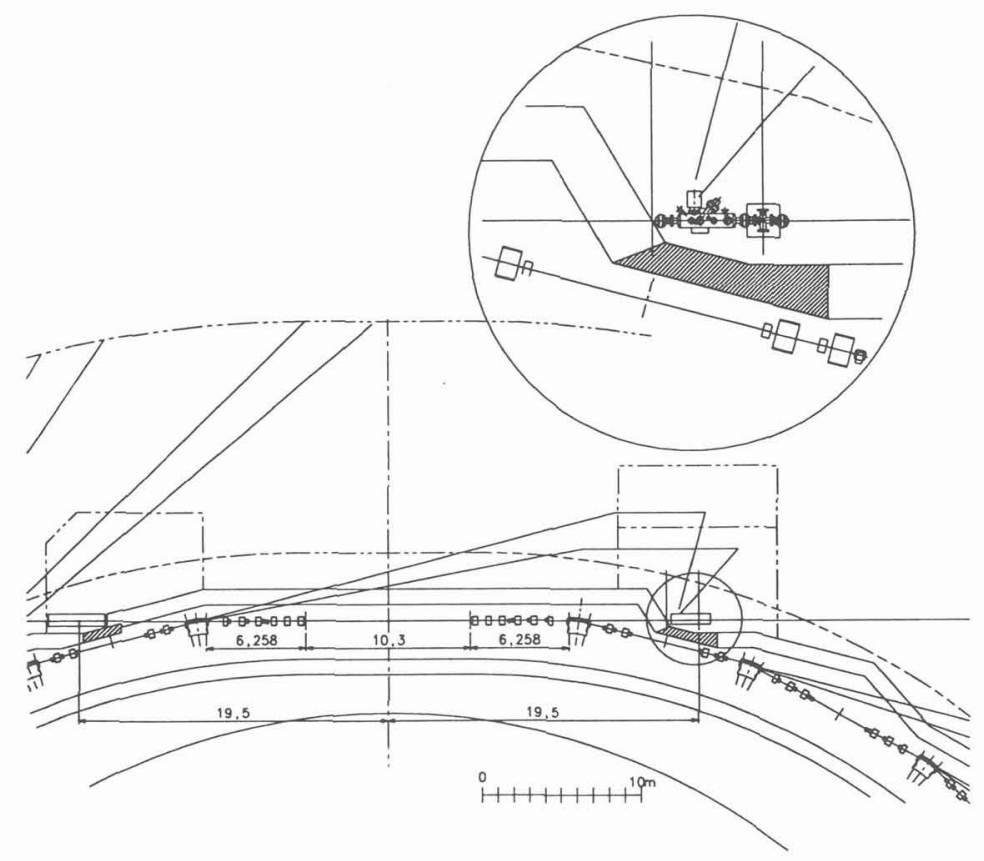

Fig. 6 : FEL straight section.

\section{LIFETIME}

For the computation of the lifetime, the pressure is assumed to be 1 ntorr for $300 \mathrm{~mA}$ and $0.3 \mathrm{ntorr}$ for $80 \mathrm{~mA}$, which seems to be realistic after one year of operation.

For the Touschek lifetime we assume a (conservative) value of $1.8 \%$ energy acceptance taking into account the possible limitation due to the insertion non linear fields. The results are given also with the energy acceptance allowed by the $3 \mathrm{MV}$ RF voltage limited to a maximum value of $3.5 \%$. For the different modes of operation they are :

Table 2

\begin{tabular}{|c|c|c|c|c|c|}
\hline & $\mathrm{E}=2.15 \mathrm{G}$ & & $=300 \mathrm{~mA}$ & $\begin{array}{r}\mathrm{E}=2.15 \mathrm{GeV} \\
\mathrm{I}=8 \times 10 \mathrm{~mA}\end{array}$ & $\begin{array}{r}\mathrm{E}=1.5 \mathrm{GeV} \\
\mathrm{I}=4 \times 5 \mathrm{~mA}\end{array}$ \\
\hline$\varepsilon_{\mathrm{x}}(\mathrm{nm} \cdot \mathrm{rad})$ & 30 & 11 & 3.9 & 30 & 16 \\
\hline$\varepsilon_{\varepsilon}(\%)$ & 1.8 & 1.8 & 1.8 & 1.8 & 1.8 \\
\hline$\sigma_{\ell}(\mathrm{mm})$ & 6.6 & 6.6 & 6 & 6.6 & 5.4 \\
\hline$\tau(\mathrm{h})^{*}$ & 24 & 16 & 10 & 10 & 5.2 \\
\hline $\begin{array}{l}\text { V (MV) } \\
\text { for } \varepsilon_{\varepsilon}=3.5 \%\end{array}$ & 3 & 3 & 2.2 & 3 & 1.74 \\
\hline$\sigma_{\ell}(\mathrm{mm})$ & 4.1 & 4.1 & 3.9 & 4.1 & 3.1 \\
\hline$\tau(h)^{*}$ & 30 & 23 & 18 & 29 & 20 \\
\hline
\end{tabular}

* These calculations are given with $\kappa^{2}=0.1$ except for the case $\mathrm{E}=1.5 \mathrm{GeV}$ where $\mathrm{k}^{2}=1$. 


\section{TECHNICAL CHOICES}

\subsection{Injector}

The full energy injection means no ramping and guarantees good beam position reproducibility. Furthermore high intensity instabilities which are more severe at lower energy are avoided. Although needing an extra capital and operation cost for the preinjector $(\approx 40 \mathrm{MFF})$ the positron option is safer from the point of view of stability and lifetime as it is shown below.

Ion trapping in the electrons beam increases the beam size and spoils the stability and lifetime. This effect can be minimized by leaving a gap in the bunch train instead of a uniform filling and reducing coupling between vertical and horizontal emittance $\left(\kappa^{2}\right)$. Unfortunately, for medium energy range the density per bunch increasing, the Touschek lifetime decreases. For example, $\varepsilon_{x}=1110^{-9} \mathrm{~m} \cdot \mathrm{rad}$ and $300 \mathrm{~mA}$ :

$\begin{array}{lll}\text { - Uniform filling } & \kappa^{2}=0.1 & \tau=16 \mathrm{~h} \\ \text { - 5/7 filling } & \kappa^{2}=0.01 & \tau=8 \mathrm{~h}\end{array}$

In many cases of machines operating with electron, ion trapping effect failed to explain the observed events, another process has been proposed [4], related to the trapping of macro particles (dust of all kind) photoionized by synchrotron radiation. They are raised into the beam, then further ionized by the electrons and finally trapped in the beam potential. It results in sudden losses and/or bad lifetime.

Furthermore, beam losses or lifetime accidents are observed from various synchrotron radiation sources operating with electrons when insertion device gaps are changed* ${ }^{*}$. No observation of this kind was so far reported from machines operating with positron beams.

Presently, ALS (Berkeley) and ELETTRA (Trieste), are in commissioning process and we expect results about electron beam behaviour in the above conditions, especially in single or few bunch operation mode.

\subsection{Cavity option}

Coherent multibunch instabilities driven by the higher order modes (HOM) of the cavities are one of the intensity limitations for the storage ring synchrotron radiation sources. The longitudinal and transverse stability for multibunch operation is especially important for SOLEIL and all the projects which have non zero dispersion in the straight sections equipped with insertion devices. With the pessimistic assumption that the parasitic frequency of the cavity is exactly on the synchrotron dipole side band, the threshold of the longitudinal oscillation is approximately given by :

$$
\mathrm{I}_{\mathrm{TH}} \approx \frac{4 \pi\left(\mathrm{E}_{\mathrm{O}} / \mathrm{e}\right) \mathrm{v}_{\mathrm{S}}}{\tau_{\mathrm{E}} \alpha} \times \frac{1}{\omega_{\mathrm{T}} \mathrm{R}_{\mathrm{S}}}
$$

where $E_{O}$ is the particle energy, $\nu_{\mathrm{S}}$ the synchrotron tune, $\alpha$ the momentum compaction factor, $\tau_{\mathrm{E}}$ the damping time, $\omega_{\mathrm{I}}$ and $\mathrm{R}_{\mathrm{S}}$ respectively the cavity HOM frequency and its shunt impedance.

For low or medium energy range machine and rather low periodicity, the higher order modes are severe. Studies have been performed with different kinds of cavities and it turns out that a "monomode" cavity will be a worthwhile option for SOLEIL.

The solution of superconducting CORNELL cavity [5] $(500 \mathrm{MHz})$ is considered and LURE participates in the development studies. The parameters are chosen as follows :

$\mathrm{U}_{\mathrm{o}}=450 \mathrm{keV}$ with insertions

\footnotetext{
* Note that at the ESRF the phenomenom has been explained by the migration of magnetic particles out of the beam path during the dynamic change of the magnetic field and not related to the electron or positron behavior.
} 


$$
\begin{aligned}
& \mathrm{I}_{\text {Beam }}=500 \mathrm{~mA} \\
& \mathrm{~V}_{\mathrm{RF}}=3 \mathrm{MV}
\end{aligned}
$$

$\mathrm{P}_{\text {Beam }}=225 \mathrm{~kW}$

2 cavities fed by 4 klystrons $(75 \mathrm{~kW})$.

\subsection{Stability requirements}

The user requirements for the photon beam stability are the following :

$-1 \mu \mathrm{m}$ over 1 hour (instantaneous),

$-10 \mu \mathrm{m}$ over 1 day (short term),

- $100 \mu \mathrm{m}$ over long term (between alignments).

These constraints are converted in following civil engineering criteria :

Table 3

\begin{tabular}{|c|l|l|}
\hline Criterium & \multicolumn{1}{|c|}{ Stress } & \multicolumn{1}{c|}{ Maximum displacement } \\
\hline Static & $500 \mathrm{~kg}$ load & $6 \mu \mathrm{m}$ upright the load \\
& & $2 \mu \mathrm{m}-2 \mathrm{~m}$ from the load \\
& $80 \mathrm{~kg}$ moving mass & $1 \mu \mathrm{m}$ and 0.3 respectively \\
\hline Dynamic & Vibrations $: 0.1 \rightarrow 70 \mathrm{~Hz}$ & $1 \mu \mathrm{m}$ peak to peak \\
\hline
\end{tabular}

A study has been performed by the SETEC company. The results are applicable to the reference site : "L'Orme des Merisiers" on the "Plateau de Saclay".

With regard to the foundations, two solutions have been considered, taking into account the cultural and technical noises. One slab laying on two meter thick artificial substrat meets the previous requirements and seems better than the second solution "jacketed piles" which is too sensitive to horizontal stresses. The slab is 1 meter thick with honey comb pattern for the experimental hall and 1.20 meter thick for the machine and its shielding. The metallic structure of the hall and the two slabs are independent.

In order to meet the beam stability requirements, girders are used as quadrupole multiplets support. Temperature control is mandatory (machine $\pm 0.2^{\circ} \mathrm{C}$, experiments $\pm 1{ }^{\circ} \mathrm{C}$ ) and closed orbit feedback has to be implemented to combat the lattice amplification factor $\left(A_{x}=60, A_{z}=30\right)$. It consists of a harmonic global feedback using Beam Position Monitor (BPM) of the machine and a local feedback for insertion devices based on photon detectors or BPM. HLS system as developped at ESRF has to be installed on both the storage ring and the beamlines.

\section{CONCLUSION}

The study of the flexible version of SOLEIL looks very promising but is not yet completed. More optimisation is required on optics studies. An alternative solution replacing four bending magnets by superconducting dipoles is also under consideration, being more attractive than the implementation of wigglers.

\section{AKNOWLEDGMENT}

We thank all the LURE technical groups who were involved in this project and the LNS staff for the study of the booster and beam transports. We are specially indebted to their theoretical study group which collaborates to the present storage ring optics study. 


\section{REFERENCES :}

[1] Workshop Proceedings, "Review of Target Specifications for Storage Ring Synchrotron Radiation Light Sources \& Means of achieving them", ESRF, Grenoble, October 19-20, 1993.

[2] LURE Internal Report, "Etude Technique SOLEIL", January 1994.

[3] Couprie M.-E., Billardon M., "The Super-ACO free electron laser in the UV, first application for time-resolved fluorescence in biology and prospects for SOLEIL", this conference.

[4] Marin P., "Problems encountered with stray micro-objects in various ultra-high vacuum systems", Anneaux RT/93-04.

[5] Padamsee $H$. et al., "Superconducting RF accelerating and crab cavities for the Cornell B-Factory, CESR-B", CNLS 90-1039. 